Chapter 4

\title{
Diagnostic Use of Sonography in the Evaluation of Hypertension
}

\author{
Nikolaos Pagonas, Stergios Vlatsas and \\ Timm H. Westhoff \\ Additional information is available at the end of the chapter \\ http://dx.doi.org/10.5772/56171
}

\section{Introduction}

Hypertension is the most frequently treated disease in internal medicine. More than 1 billion people worldwide suffer from hypertension. Hypertension leads to cardiovascular end-organ damage increasing morbidity and mortality and is related with high costs to society, making this disease an important public health challenge. Sonography is a crucial diagnostic tool in the evaluation of a hypertensive patient. It is used both for the search of secondary forms of hypertension and for the identification of hypertensive end organ damage. There are several ultrasound examinations that may be warranted in hypertension. Abdominal ultrasound is recommended by several guidelines for the basic diagnostic workup in every newly diagnosed hypertensive patient. Doppler sonography of the renal arteries is reasonable only in a subset of hypertensives that are at increased risk of renal artery stenosis. Echocardiography is able to reveal cardiac end organ damage in terms of hypertensive heart disease. Ultrasound of the carotid arteries is frequently used to detect and evaluate in the case of hypertension-induced vascular end organ damage. The assessment of the intima-media thickness allows the detection of early stages of atherosclerotic wall changes. Prior to any structural vascular damage that may be visualized by ultrasound techniques, hypertension leads to functional changes of the endothelium, called endothelial dysfunction. Endothelial dysfunction encompasses a variety of changes in vascular function including a reduced endothelium-dependent vasodilation. This can be diagnosed by sonography measuring the diameter changes of the brachial artery in response to predefined endothelial stimuli. Flow-mediated dilation in response to hyperemia is regarded as the gold-standard in the non-invasive assessment of endothelial dysfunction. To date, it is rather used scientifically than in daily clinical practice. The present chapter provides 
an overview on the practical performance of all of these ultrasound techniques in the approach to hypertension.

\section{Abdominal ultrasound}

The use of abdominal ultrasound in the evaluation of hypertension is twofold.

- In the detection of a secondary forms of hypertension.

- In the evaluation of subclinical organ damage induced by hypertension.

In the current European Society of Cardiology/European Society of Hypertension (ESC/ESH ) guidelines for hypertension the use of abdominal ultrasound is recommended as a part of the evaluation of hypertensive individuals. The abdominal ultrasound supplies information about the etiology of hypertension as well as possible end organ damages.

The main interest is the morphology of the kidneys, the adrenal glands and of the aorta. Due to their retroperitoneal position, kidneys are completely and easily detectable. A $3.5-5 \mathrm{MHz}$ probe is typically used to scan the kidney. The examination from dorsolateral allows the evasion of the intestinal loops and thus allows for a non-overlapping imaging in the supine position. The formerly widely spread examination in the lateral recumbent position is nowadays used only in exceptional cases. Renal ultrasound has now almost completely replaced intravenous urography in the anatomical exploration of the kidney. While the latter requires the injection of potentially nephrotoxic contrast medium, ultrasound is non-invasive and provides the necessary anatomic data about kidney size and shape, cortical thickness, urinary tract obstruction and renal masses [1]. Renal parenchymal disease is one of the most common causes of secondary hypertension which leads to a wide spectrum of morphologic alterations. The finding of bilateral upper abdominal masses at physical examination is consistent with polycystic kidney disease and should warrant an abdominal ultrasound examination. Acute parenchymal inflammatory processes like crescentic glomerulonephritis or acute interstitial nephritis sometimes predisposes individuals to measurable organ swelling. The cortical and medullary pyramids have in this case an anechoic profile. However, the morphological alterations seen via ultrasound in the acute kidney processes are less prominent than those seen in chronic kidney damage. Thus, the diagnostic performance in acute inflammatory renal disease is less effective. Chronic parenchymal diseases, such as chronic interstitial nephritis, glomerulonephritis or nephrosclerosis contribute to a progressive decrease in organ size. A kidney size below $90 \mathrm{~mm}$ should be interpreted as pathological. Over the course of the organ decrease, small scarring cortical retractions develop, which give the kidney surface a humped aspect. The renal parenchyma develops a hyperechoic pattern through progressive scarring. The border between parenchyma and pyelon becomes progressively nondescript. A variety of chronic parenchymal diseases can lead to the morphological end stage of a shrunken kidney. Sonographically, it is not possible to differentiate whether small kidneys are the cause or the result of hypertension. A unilateral small kidney as a possible indicator for a hemodynamic relevant renal artery stenosis should always lead to a further evaluation of the renal 
arteries. Renal cell carcinomas as a rare cause of hypertension are depicted as a well delimitable structure from the surrounding tissue. Usually they can be depicted via ultrasound when they exceed $1 \mathrm{~cm}$. With increasingly size there is an increase in their inhomogeneity, so that it is possible to detect areas of liquefied necrosis for example.

In the screening of secondary forms of hypertension abdominal ultrasound plays also a role in the depiction of the adrenal glands. For this purpose a detailed knowledge of the local anatomy is required. The adrenal glands are located within the retroperitoneum. The left adrenal gland, lacking the acoustic window of the liver and being obscured by air in the stomach, is inherently more difficult to scan than the right adrenal gland. On the right side, the right kidney and the inferior vena cava are landmarks for the examination of adrenal glands, whereas on the left side the aorta, the lower pole of the spleen and the upper pole of the kidney are points of orientation. The right adrenal gland is usually scanned with a right transcostal scan or a subcostal flank scan or oblique subcostal scan. On the left side it is better to use an intercostal flank scan through the spleen. The normal sized adrenal glands are only visible with trained examination techniques and by using high resolution technology, whereas enlarged adrenal glands are detectable in a high percentage of cases. Thirty percent of cases of primary aldosteronism are caused by adrenal adenomas. Seventy percent of cases are caused by adrenal hyperplasia. There are rare cases of adrenal carcinoma and the autosomal dominant condition of glucocorticoid remediable aldosteronism [2]. The micronodular hyperplasia is not possible to be detected via sonography. Adrenal adenomas have a round to oval shape and are uniformly hypoechoic with smooth margins, although some lesions have scalloped borders (polycyclic). Adenomas occasionally have an inhomogeneous appearance. Autopsy statistics indicate that they are quite common (10-20\%), but most adenomas (90\%) produce no endocrine symptoms, they are silent and too small to be detected by ultrasound. In one study the average size of adenomas was reported to be $1.5 \mathrm{~cm}$, although they may exceed $5 \mathrm{~cm}$ in diameter. In a small percentage of patients adenomas are bilateral. Functioning and nonfunctioning adenomas are indistinguishable by their sonographic features [3]. Thus, ultrasound is not a sufficient test in the morphologic diagnosis of Conn syndrome. Upon the detection of a high aldosteroneto-renin ratio and after a confirmation test (e.g. suppression after administration of sodium chloride) the use of a CT or MRT scan is indicated. On the other hand, the detection of a unilateral adrenal mass seen in the ultrasound should be followed by a laboratory evaluation for the evaluation of Conn-Syndrome.

Phaeochromocytoma, a tumor of the adrenal medulla, is a rare secondary cause of hypertension $(0.2-0.4 \%$ of all cases of elevated blood pressure) with an estimated annual incidence of $2-8$ per million population.[4]. It can be inherited or acquired. Hypertension occurs in about $70 \%$ of all cases of phaeochromocytoma, being stable or paroxysmal in approximately equal proportions. The diagnosis is based on establishing an increase in plasma or urinary catecholamines or their metabolites (e.g. (nor-) metanephrines). Following the appearance of clinical symptoms (hypertension and tachycardia caused by increased catecholamine secretion), pheochromocytoma can be detected in $80-90 \%$ of cases via abdominal ultrasound. Most pheochromocytomas are already several centimeters in diameter when diagnosed. They have smooth margins, a round shape, and an inhomogeneous or complex echo structure. Hypoe- 
choic liquid components may also be observed. A spectrum of appearances is possible. Pheochromocytomas are bilateral in approximately $10 \%$ of cases and extra-adrenal in $10-20 \%$. The organ of Zuckerkandl should be looked for at the level of the origin of the inferior mesenteric artery, anterior to the aorta. Other extra-adrenal sites are the renal hilum, bladder wall, and thorax. Pheochromocytoma is occasionally seen posterior to the renal vein in transverse scans. Rarely, pheochromocytoma is diagnosed in the setting of multiple endocrine neoplasia (MEN). About $2 \%$ to $5 \%$ of pheochromocytomas are malignant. In recent years endosopic sonography is being used to obtain an adrenal gland biopsy [5-7].

Abdominal ultrasound is also being used in the evaluation of hypertension induced end-organ damage. Vascular end-organ damage may be visualized as atherosclerotic as well as aneurysmatic wall alterations, e.g. of the aorta. In the elderly (> 65 years) approximately $60 \%$ of the patients with hypertension have an isolated systolic hypertension. This is a result of the diminished elasticity of the large arterial vessels. Ultrasound can indicate a morphological correlate in form of a manifest aortosclerosis. Besides vascular end-organ damage abdominal ultrasound detects renal end organ damage. The correlate of hypertensive end-organ damage of the kidney is (benign) nephrosclerosis. The sonographic features include a reduced size, hyperechoic parenchyma, indefinite margin of parenchyma and pyelon, and scarring cortical retractions. As stated above, this unspecific sonographic appearance does unfortunately not allow a differentiation between cause and result of hypertension.

\section{Echocardiography}

Hypertension is one of the most important risk factors for heart failure with increasing risk in all age groups. The lifetime risk for developing heart failure is doubled for subjects with blood pressure $>160 / 100 \mathrm{~mm} \mathrm{Hg}$ compared to those with blood pressure $<140 / 90 \mathrm{~mm} \mathrm{Hg}$ [8]. Systolic and diastolic heart failure are both associated with hypertension. There are several mechanisms, alone or in combination, leading to development of heart failure in the presence of hypertension: left ventricular hypertrophy $(\mathrm{LVH})$, chamber remodeling, hemodynamic load and coronary microvascular disease with impaired coronary hemodynamics. To assess subclinical organ damage, such as ventricular hypertrophy, echocardiography is more sensitive than electrocardiography [9], which is a routine examination in all subjects with high blood pressure. However, the ESC/ESH guidelines suggest that in patients with low and intermediate cardiovascular risk an echocardiography should be performed for better global cardiovascular risk stratification which may implicate the appropriate pharmacological treatment [10]. The role of echocardiography is not limited to identification of (sub-) clinical organ damage in the pre-treatment phase. Since changes of the left ventricular hypertrophy in response to treatment are associated to cardiovascular fatal and non-fatal events [11], echocardiography can also be used to monitor treatment's success and re-assess overall risk.

Left ventricular hypertrophy is the first step toward the development of hypertensive heart disease. The echocardiographic evaluation of LVH includes measurements of the interventricular septum, left ventricular posterior wall thickness and end-diastolic diameter. Upon 
these parameters obtained by M-Mode at the end of diastole (under two-dimensional control), the left ventricular mass is calculated according to the proposed formula [12]. Since LV mass is depended on gender and obesity, the thresholds for presence of LVH mass are indexed to body surface area and estimated for men (above $\left.125 \mathrm{~g} / \mathrm{m}^{2}\right)$ and for women (above $\left.110 \mathrm{~g} / \mathrm{m}^{2}\right)$ [10]. The adaptation of the left ventricle to hypertension is heterogenic and can be classified in three geometric patterns based on the LV mass and on the index of relative wall thickness (LV wall thickness / chamber radius). An increased ratio $\geq 0.42$ combined with increased mass is referred to as concentric hypertrophy. The term eccentric hypertrophy refers to subjects with normal wall to radius ratio $(<0.42)$ but increased LV mass. The last pattern, the concentric remodeling, refers to subjects with normal ventricular mass but increased ratio $(\geq 0.42)$. All three types of chamber remodeling in response to hypertension are related to increased cardiovascular risk. Interestingly, the incidence of cardiovascular events correlates with changes in geometric adaptation, independent of changes of the LV mass. The development or the persistence of a concentric geometry during treatment has been found to be associated with a greater incidence of cardiovascular events [13]. In the Losartan Intervention For Endpoint reduction in hypertension (LIFE) study [11] a regression of the left ventricular mass of about $25 \mathrm{~g} / \mathrm{m}^{2}$ was associated with a $20 \%$ reduction in the incidence of the primary endpoint (cardiovascular mortality, myocardial infarction, stroke). Recent data have furthermore reinforced the predictive value of echocardiography in hypertensive patients. 35.000 normotensive and hypertensive participants with normal left ventricular ejection fraction were studied retrospectively. An abnormal left ventricular geometric pattern was found in $46 \%$ of the patients (35\% with left ventricular concentric remodeling and $11 \%$ with $\mathrm{LVH}$ ) and was associated with a double-risk of all-cause mortality compared to the patients with normal left ventricular geometry [14]. A prospective trial showed that hypertensive patients with echocardiographic LVH had significantly higher all-cause mortality and cardiovascular events [15]. Beyond the lower incidence of cardiovascular events, including sudden death, in patients with regression of echocardiographic left ventricular hypertrophy or a delayed increase in left ventricular mass [11], treatment-induced changes of left atrium dimension and ventricular geometry are also correlated with cardiovascular event rates $[16,17]$.

Even before evidence of left ventricular hypertrophy is present and before hypertension in young normotensive male offspring of hypertensive parents has developed, diastolic dysfunction may develop as an early end organ damage due to hypertension [18]. Patients with diastolic heart failure (also referred as heart failure with preserved ejection fraction) show similar long term impairments as patients with systolic heart failure [19]. The importance of an early recognition of diastolic dysfunction is imperative. Arterial hypertension with or without hypertrophy is the main cause of diastolic dysfunction, namely the inability of the heart to adequately fill with blood during diastole. There are several factors which lead to diastolic dysfunction in hypertension by impeding the active or passive phases of diastole. Of these, contractile alterations in myocytes, structural ventricular hypertrophy, extracellular and perivascular fibrosis, and myocardial ischemia are most often implicated. The European Society of Cardiology has recognized diastolic dysfunction diagnosed by echocardiography as criterion for the diagnosis of diastolic heart failure [20]. There are a number of specific echocardiographic indicators of diastolic dysfunction obtained during the examination. The 
major four parameters include transmitral Doppler inflow velocity patterns, pulmonary venous Doppler flow patterns, tissue Doppler velocities and color M-mode flow propagation velocity (Vp). Transmitral Doppler flow is acquired by placing a pulsed wave (PW) sample volume at the level of the tips of the mitral leaflets in the apical four-chamber view. Normally, the ventricular inflow consists of an early (E) and a late filling peak (A). Respectively, the early filling peak velocity (E) and the late (atrial) peak velocity (A) should be recorded. In normal young individuals, more forward flow occurs during the early diastole largely due to the rapid decline in left ventricular pressure during the isovolumetric relaxation time (IVRT). Consequently, the E/A ratio is $>1$ correlating with a normal relaxation. An E/A ratio $<1$ together with prolonged IVRT and deceleration time (DT, rapid decline of the E) indicate an abnormal relaxation. By considering these three parameters two more patterns of impaired diastolic function are known: the "pseudonormal" pattern which turns to an impaired relaxation pattern when a Valsalva maneuver is performed and the restrictive pattern. The last one, occurring mostly in patients with restrictive cardiomyopathies (e.g., infiltrative sarcoidosis, endomyocardial fibrosis) and dilated cardiomyopathies with poor systolic function which is associated with increased mortality [21]. Unfortunately, mitral flow is influenced not only by the diastolic properties of the LV but also by other factors, including preload, afterload, heart rate and the presence of arrhythmias. Another indicator, the pulmonary venous flow can be used to assess diastolic function but it is also limited in case of mitral valve disease, heart block and tachycardia. A more precise assessment of the diastolic function can be made by using the tissue Doppler imaging (TDI). This enables the measurement of frequency Doppler shifts caused by myocardial motion as the mitral annulus recoils back toward the base in early (e') and late (a') diastole. The peak waves ( $\mathrm{e}^{\prime}$ and $\left.\mathrm{a}^{\prime}\right)$ are obtained in analogy to those recorded by the mitral flow. TDI enables, depending of the placement of the sample volume, assessment of global or regional diastolic function. In patients with hypertension and hypertrophy, diastolic dysfunction is more evident at the basal septal segments [22]. The E/e' ratio (with e' assessed at a lateral segment) has been identified as the best parameter for diagnosis of diastolic heart failure [23]. An E/e' ratio below 8 is associated with normal filling pressures and a ratio $>12$ to 15 is associated with elevated filling pressures. For values between 8 and 12 additional echocardiographic parameters (e.g., use of Valsalva with transmitral Doppler, pulmonary venous flow) are recommended to correctly classify diastolic function. By using the Doppler color M-mode another index of diastolic dysfunction, the propagation velocity of early diastolic flow $(\mathrm{Vp})$ into the ventricle has been proposed. This index seems to be independent of the load conditions and can be useful to unmask diastolic dysfunction in hypertensive patients with pseudonormal mitral flow. However, in patients with normal left ventricular function the Vp may be normal despite an impaired left ventricular relaxation indicating a major limitation of the index. Beside diastolic dysfunction, an enlarged left atrium was found in patients with hypertension and preserved ejection fraction and is associated with elevated filling pressures of the left ventricle leading to clinical heart failure [24].

As described earlier, echocardiography assesses two main features of the hypertensive heart disease, left ventricular hypertrophy and diastolic dysfunction. Systolic dysfunction occurring in the presence or not of the aforementioned changes is assessed in the clinical practice by echocardiography. Assessment of the ejection fraction can be made visually, it requires 
however a high level of expertise and is limited by subjectivity. Quantified, objective measurements of the LV systolic function have become standard practice in echocardiography. One parameters of the systolic function is fractional shortening obtained from M-Mode tracings in the parasternal long axis (method according to Teichmann). Though it is a simple and quick method, it is limited by the fact that it provides information about contractility along a single line. If regional wall motion abnormalities occur (e.g. in the presence of coronary artery disease) the severity of the dysfunction may be under - or overestimated, depending if the region of an abnormal wall motion is interrogated or not. As long as this method is only valid in symmetrically contracting hearts, it is inappropriate for the remodeled ventricles of patients with heart failure. The European and American guidelines recommended the biplane method of discs (modified Simpson's rule) as the echocardiographic method of choice for volume measurements and estimation of ejection fraction $[25,26]$. The principle underlying this method is that the total LV volume is calculated from the summation of a stack of elliptical discs. The height of each disc is calculated as a fraction of the LV long axis based on the longer of the two lengths from the two and four-chamber views. The cross-sectional area of the disk is based on the two diameters obtained from the two- and four-chamber views. The method can also be used with one single plane, when two orthogonal views are not available. In this case, the presence of any extensive wall motion abnormalities may limit the results [25]. Practically, the endocardial borders in the apical 4- and 2-chamber views in end-diastole and end-systole are traced manually or automatically. The end-diastolic and end-systolic volumes (EDV, ESV) are calculated and the ejection fraction is estimated as follows: Ejection fraction $=(E D V-E S V) /$ EDV. The reference values for the ejection fraction do not differ between men und women. An $\mathrm{EF}>55 \%$ indicates a normal systolic function. An EF between $45-54 \%$ suggests a mildly abnormal function and an EF between $30-44 \%$ a moderately abnormal systolic function. A severely abnormal left ventricular function is indicated by an $\mathrm{EF}<30 \%$. This 2 -D approach to assess EF is based on geometric assumptions, which are invalid in a nonsymmetrical contracting, remodeled ventricle. Over the last decade, several three-dimensional (3-D) echocardiographic techniques became available to measure LV volumes and mass. 3-D echocardiography does not rely on geometric assumptions for volume/mass calculations and is not subject to plane positioning errors, which can lead to chamber foreshortening. Compared to the goldstandard for assessment of left ventricular volumes and EF, the cardiac magnetic resonance, 3-D echocardiography showed significantly better agreement (smaller bias), lower scatter and lower intra- and inter-observer variability than 2-D echocardiography [27, 28]. Furthermore, 3-D echocardiography is also used in the assessment of diastolic function, as it is independent of load conditions.

In summary, echocardiography is a necessary diagnostic tool for risk stratification of patients with hypertension before treatment but also for follow-up assessment of end-organ damages during treatment. In $25-30 \%$ of hypertensive patients with low or moderate cardiovascular risk (based on risk factor evaluation and ECG), an increase of the LV mass may be identified by echocardiography leading to higher risk stratification and changes of therapeutic strategy $[29,30]$. 


\section{Doppler ultrasound of renal arteries}

Renovascular hypertension is the second most common cause of secondary hypertension in approximately $2 \%$ of adult patients who present with blood pressure elevation when assessed in specialized centres [31]. This is caused by one or more stenoses of the extrarenal arteries, which in the elderly population have frequently an atherosclerotic nature. Fibromuscular dysplasia accounts for up to $25 \%$ of total cases and is the most common variety in young adults. Unfortunately there is not any optimal screening test available for the time. A screening test should have a high sensitivity in order to keep the false negative results in the lowest possible level. The Doppler ultrasound of renal arteries allows an analysis of the renal perfusion. According to various studies the sensitivity and specificity of Doppler ultrasound in the diagnosis of renal artery stenosis lies approximately at $90 \%[32,33]$. Due to the fact that renovascular hypertension in individuals with mild and medium hypertension has a prevalence below $1 \%$, an unselective examination of all individuals with hypertension would lead to a high rate of false positive results. That would result to a high rate of unnecessary angiographies. On the other hand in a preselected population of hypertensives with clinical implications of increased probability of renal artery stenosis the Doppler ultrasound of the renal arteries is a very appropriate screening examination, due to the fact that the prevalence of renovascular hypertension in acute, severe and resistant hypertension is significantly higher $(10-45 \%)$. In other words, the use of Doppler ultrasound as a diagnostic tool in a selected population, has a significantly higher positive predictive value with a still acceptable negative predictive value.

Clinical signs for a renovascular hypertension include the following:

- Hypertension in individuals younger than 30 years of age

- A unilateral small kidney or a difference in renal size more than $1.5 \mathrm{~cm}$

- Generalized atherosclerosis

- Abdominal bruit with lateralization,

- Resistant hypertension, defined as hypertension refractory to treatment with at least three antihypertensive drugs (including a diuretic agent)

- An elevation of the serum creatinine level $>30 \%$ under the treatment with an ACE inhibitor or an AT1-receptor antagonist

It should be mentioned, that a difference of more than $1.5 \mathrm{~cm}$ in length between the two kidneys, which is usually considered as being diagnostic for renal artery stenosis is only found in $60-70 \%$ of the patients with renovascular hypertension [34].

The examination itself can be limited by factors such as bowel gas, obesity, cooperation of the patient and from the fact that it is a highly operator dependent examination. The atherosclerotic induced renal artery stenosis is easily detectable, because it usually involves the ostium and the proximal $1 / 3$ of the renal artery. On the other hand fibromuscular dysplasia involves the distal $2 / 3$ of renal artery and their segmental branches and is thus difficult to depict. With 
today's ultrasound technology it is possible to visualize approximately $88 \%$ of all main and accessory renal arteries [35]. In every renal artery angle corrected flow velocity measurements should be performed in at least 5 points. A velocity of $60-100 \mathrm{~cm} / \mathrm{s}$ is considered as normal, whereas a $70 \%$ stenosis leads to velocities of at least $180-200 \mathrm{~cm} / \mathrm{s}$. Stenoses proximal to the ostium are easily obtainable with an epigastric transverse scan and distal stenoses or stenoses of segmental branches are better seen with a longitudinal flank scan (lateral position).

Additional intrarenal scanning permits the diagnosis of renal artery stenosis without direct imaging of the main renal artery. In 1994, Schwerk et al. introduced the Resistive Index (RI) obtained in the interlobar arteries as a reliable indirect parameter for detecting renal artery stenosis. The RI is a ratio of peak systolic and end diastolic velocity, derived from the Doppler spectrum of any vessel. The authors calculated the side-to-side difference of intrarenal RI $>5 \%$ with the lower RI in the post-stenotic kidney. Sensitivity and specificity were $100 \%$ and $94 \%$, respectively, for moderate and severe RAS [36]. In the meantime, intrarenal RI has been frequently evaluated for different nephrological issues [37, 38]. In a single prospective study a high intrarenal RI was found to be negatively correlated with the outcome of intervention in patients with atherosclerotic renal artery stenosis [39]. A high RI $(R I \geq 80)$ was felt to reflect advanced renal damage, which would explain the interventional treatment failure. Radermacher et al. [39] investigated the efficacy of angioplasty of a renal artery stenosis in dependence of the RI. They concluded that an increased renal resistance index $>0.8$ is associated with a poor prognosis despite correction of the stenosis. To date, the clinical impacts of these findings are discussed controversially.

\section{Ultrasound of carotid arteries}

The carotid wall thickening is an early marker of atherosclerosis and subclinical organ damage. It precedes the evolution of arteriosclerotic plaques. Ultrasound of the carotid arteries constitutes a very good opportunity to evaluate hypertension-induced vascular end organ damage. Several cardiovascular risk factors including male sex, ageing, elevated blood pressure, diabetes, smoking and obesity are positively associated with increased carotid intima-media thickness (IMT) in observational and epidemiological studies. Above these factors, high systolic blood pressures have the greatest effect on IMT [40]. An IMT >0.9 mm in the common carotid artery is generally seen as abnormal; however there is a continuous relationship between IMT and cardiovascular events. An intima-media thickness (IMT) $>0.9$ $\mathrm{mm}$ or the presence of a carotid plaque predict the occurrence of stroke and myocardial infarction $[2,41]$. Particularly, in a meta-analysis of data from 8 studies in general populations, including about 37,000 subjects who were followed up for a mean of 5.5 years, the risk for a myocardial infarction increases by $10-15 \%$ and the stroke risk by $13-18 \%$ for every $0.1 \mathrm{~mm}$ increase of the IMT [42]. For the assessment of CVD risk, the carotid artery wall, rather than the degree of luminal narrowing, is examined to identify areas of increased thickness and nonocclusive atherosclerotic plaque, which represent early stages of arterial injury and atherosclerosis. Furthermore, the detection of early signs of vascular damage has to include ultrasound not only of the common carotid arteries, but of bifurcations and/or internal carotids 
where atherosclerosis progresses more rapidly and plaques are more frequent [43]. Ultrasound imaging of the far wall of the carotid artery produces two echogenic lines, which correspond to the lumen-intima interface and the media-adventitia interface. The current ultrasound technology enables in the clinical practice the combined measurement of the thickness of the intimal and medial layers of the arterial wall which constitute the IMT. Carotid plaque is defined as the presence of focal wall thickening that is at least $50 \%$ greater than that of the surrounding vessel wall or as a focal region with IMT greater than $1.5 \mathrm{~mm}$ that protrudes into the lumen. Both near and far walls can be used for assessment of the IMT. However, IMT of the near wall is less accurate because the ultrasound beam is traveling from more to less echogenic layers at the adventitia-media and intima-lumen interfaces of the near wall [44]. Histological data suggest an underestimation of the IMT around $20 \%$ when the near wall is used [45]. A linear-array transducer operating at a frequency of at least $7 \mathrm{MHz}$ [44]. Three methods most frequently used to measure the IMT by using B -Mode are the following: 1 . Averaging the maximum IMT of the four far walls of the carotid bifurcations and of the distal common carotid arteries. 2. Assessing the mean maximum thickness ( $\mathrm{M}$ max) of up to 12 different sites (right and left, near and far walls, distal common, bifurcation, and proximal internal carotid). 3. The maximum measured IMT of a single measurement is taken into account. The last method provides more reproducible results when IMT measurement is restricted to the far wall of the distal segment of the common carotid artery, providing only a $3 \%$ of relative difference between two successive measurements [46]. Analysis may be performed by manual cursor placement or by automated computerized edge detection. As we mentioned above, B-mode imaging is preferred over M-mode imaging. M-mode, in spite of a superior temporal resolution, provides measurement of one single point of thickness, rather than a segmental value as it is enabled by B-mode. However, carotid wall thickening is not uniform and a singe point measurement may not represent accurately the arterial status and is less reproducible for follow-up measurements. A novel noninvasive echo-tracking system measuring the IMT and other mechanical properties of the carotid wall has been proposed [47]. This method enables an additional evaluation of the carotid plaque stability and composition.

The normal IMT values are influenced by age and sex and IMT normal values may be defined in terms of statistical distribution within a healthy population. IMT values greater than the 75 th percentile are considered high and indicate an increased cardiovascular risk. Values in the 25th to 75th percentile are considered average and indicative of unchanged CVD risk. Values less than or equal to 25th percentile are associated with a low CVD risk [44]. However, available data indicate that IMT $>0.9 \mathrm{~mm}$ represents a risk of myocardial infarction and/or cerebrovascular disease and in the clinical practice this cut-off value may better defined in terms of increased risk [48]. Recent data have further strengthened the relationship of carotid IMT and plaques with cardiovascular events. A new report from the European Lacidipine Study on Atherosclerosis (ELSA) trial suggests that baseline carotid IMT predicts cardiovascular events independent of blood pressure and this occurs both for the IMT value at the carotid bifurcations and for the IMT value at the level of the common carotid artery [49]. The adverse prognostic significance of carotid plaques has also been reported in patients with high normal blood pressure prospectively followed for about 13 years [50]. In the Risk Intervention Study (RIS) study patients with severe essential hypertension and high cardiovascular risk had a 
significantly higher prevalence of atherosclerotic lesions compared to control subjects [51]. Assessment of IMT plays an important role in the risk stratification. Interestingly, about $30 \%$ of hypertensive subjects classified as at low or moderate added risk without ultrasound for carotid artery thickening or plaque were placed in the high added risk group after detecting of vascular damages [29].

The predictive value of the carotid ultrasound and its role in risk stratification has been demonstrated in a lot of trials as aforementioned. Whether a decrease of IMT progression is associated with a reduction of cardiovascular events and an improvement in prognosis remains at the time elusive. Therapeutic double blind trials have shown that antihypertensive drugs may have a more or less marked effect on carotid IMT progression. A recent metaanalysis of 22 randomized controlled trials has evaluated the effects of an antihypertensive drug versus placebo or another antihypertensive agent of a different class on carotid intimamedia thickness. The results have shown that compared with no treatment, diuretics $\$ \pm$ betablockers, ACE inhibitors and calcium antagonists attenuate the rate of progression of carotid intima-media thickening, in some trials even in the absence of any significant reduction of the blood pressure [52]. In patients with hypertension and hypercholesterolemia the administration of pravastatin prevents the progression of carotid intima-media thickness [53]. In the ELSA trial the treatment-induced changes of the IMT did not predict cardiovascular events as was the case by the baseline values. However, these results are not conclusive due to the smallness of the IMT changes and the large individual differences in baseline IMT [49].

Beyond the identification of subclinical organ damage of the carotid arteries, ultrasound is a useful tool for identification of carotid stenoses in progressive atherosclerosis. High blood pressure is a major risk factor for stroke with a correlation between elevated BP and occurrence of stroke. This correlation holds over a wide BP range, from systolic levels as low as $115 \mathrm{~mm} /$ $\mathrm{Hg}$ and diastolic levels as low as $70 \mathrm{~mm} / \mathrm{Hg}$ [54], with systolic BP having a stronger association with higher stroke risk. Elevated BP is positively associated with both ischemic and hemorrhagic stroke, with a higher association appearing in hemorrhagic stroke and secondary prevention [56]. Large vessel disease of the extracranial arteries accounts for half of the ischemic strokes.

Doppler sonography is the most common imaging study performed for the diagnosis of carotid disease. It is part of the imaging tests taking place early after a transient ischemic attack (TIA) or stroke in order to identify patients with tight symptomatic arterial stenosis who could benefit from endarterectomy or angioplasty. Carotid Doppler-studies are fast, non-invasive and easily applicable however provide limited information, require skilled operators and are investigator-dependent. Doppler ultrasound has a similar specificity and sensitivity for carotid artery stenosis with computed tomography angiography (CTA) but both are inferior to contrast-magnetic resonance angiography (MRA) [57]. For asymptomatic patients with hypertension, assessment for carotid artery stenosis is also useful as part of risk stratification and is recommended for patients with two or more risk factors for atherosclerosis. Evidence of an internal carotid artery stenosis (ICA) supports recommendation of antiplatelet therapy and more strict control of risk factors to prevent stroke [58]. 
The first step of a carotid ultrasound test is to identify plaques via B-mode as referred above. Addition of color Doppler enables identification of origin and course of the internal carotid artery and a differentiation between severe stenosis and occlusion. Stenotic areas are identified in the presence of the "aliasing" phenomenon occurring due to high velocities in the center of the stenotic lumen and post-stenotic flow disturbances. Flow velocities in the spectral analysis are the main parameters used for evaluating the severity of carotid stenosis. Flow velocity must be sampled through the whole area of presumed stenosis until the distal end of the plaque is seen to ensure that the site of the highest velocity has been detected. Compared to angiographic data, a wide range of flow velocities was recorded for any given degree of angiographic stenosis so that the sensitivity and specificity of the method may vary [59]. A better correlation to angiographic determined stenoses is achieved by assessing the peak systolic velocity in the internal carotid artery and the ratio of the peak systolic velocity in the internal carotid artery to that in the ipsilateral common carotid artery as proposed by the current guidelines [60]. Based on Doppler sonography, carotid stenoses are classified into two grades: Grade 1- with the rate of stenosis measuring between $50 \%$ to $69 \%$ and Grade 2 measuring 70-99\%, which also represents a severe non-occlusive disease. In equivocal cases, further imaging methods may be additionally used. In Table 1 the sonographic criteria for grading of carotid artery stenosis are summarized.

An ultrasound examination for detection of carotid stenoses and plaques and evaluation of the intima-media thickness should be performed in hypertensive patients with concomitant risk factors such as smoking, dyslipidemia, diabetes, obesity and family history of cardiovascular disease. The results can be useful for re-assessing CVD risk in some asymptomatic patients and consequently re-assessing therapeutic strategies. For accurate results, strict attention to quality control in image acquisition, measurement and interpretation are necessary.

\begin{tabular}{ll}
\hline Grade of Stenosis & Peak systolic velocity and visible criteria \\
\hline no stenosis & $<125 \mathrm{~cm} / \mathrm{s}$ and no visible plaque or IMT \\
\hline$<50 \%$ & $<125 \mathrm{~cm} / \mathrm{s}$ with visible plaque or IMT \\
\hline $50-69 \%$ & $125-230 \mathrm{~cm} / \mathrm{s}$ with visible plaque \\
\hline$>70 \%$ to near occlusion & $>230 \mathrm{~cm} / \mathrm{s}$ with visible plaque and lumen narrowing \\
\hline $100 \%$ & No detectable patent lumen and flow is seen \\
\hline
\end{tabular}

Table 1. Grading of internal carotid artery stenosis upon Doppler velocities and B-mode

\section{Flow mediated dilation}

Endothelial function is linked to cardiovascular risk factors and provides prognostic information for cardiovascular diseases [61]. Endothelial dysfunction is regarded as the initial step of atherosclerosis and therefore as the earliest detectable manifestation of vascular end-organ 
damage. It can be assessed using several methods, with flow-mediated dilation (FMD) being currently the gold-standard in non-invasive evaluation of endothelial dysfunction. In this ultrasound-based method brachial artery diameter is measured before and after an increase in shear stress that is induced by reactive hyperemia. When a sphygmomanometer cuff placed on the forearm or upper-arm and is inflated at $50 \mathrm{~mm} / \mathrm{Hg}$ above systolic pressure, arterial inflow is occluded causing a local ischemia and dilation of downstream arteries. Cuff deflation induces a high-flow state through the brachial artery (reactive hyperemia) to accommodate the dilated resistance vessels. The increased shear stress leads to endothelium dependent dilation of the brachial artery. FMD occurs predominantly as a result of local endothelial release of nitric oxide. Figure 1 shows the B-mode and Doppler flow images of the brachial artery of a patient with hypertension at baseline and after deflation of the cuff (hyperemia). The FMD was calculated by the equation: $\mathrm{FMD}=($ diameter hyperemia - diameter baseline $)$ * 100 / diameter baseline. A value of $8.1 \%$ suggesting a near normal value (>8\%) was found.

By using invasive and non-invasive methods impaired endothelial function has been found in uncomplicated hypertensive patients $[62,63]$. In one prospective trial a reduction of blood pressure in response to antihypertensive treatment leads to improvement of the FMD suggesting a beneficial effect of antihypertensive treatment on endothelial function [64]. Several other large trials have found relationships between endothelial dysfunction assessed by FMD and prognostic markers of cardiovascular disease and atherosclerosis [65, 66]. Although the FMD test has opened a new field in the clinical research of conduit artery endothelial biology, some practical challenges of this technique have prevented its broad use in daily clinical practice so far. The most important of these are the need for highly trained operators, the timeconsuming analysis of results and the care required to minimize environmental or physiological influences such as eating, caffeine ingestion and variations of temperature [67].

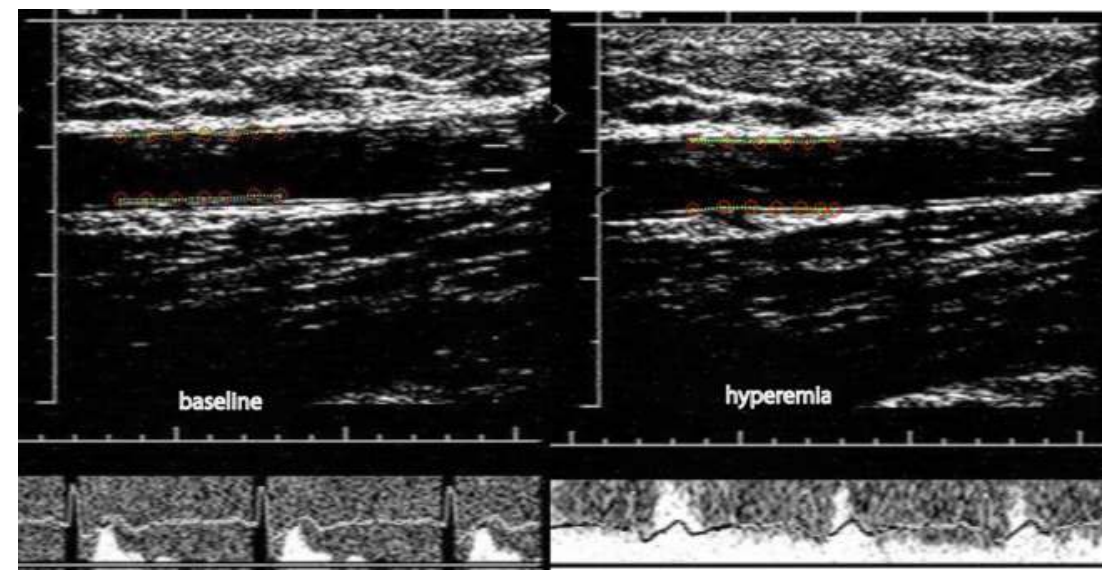

Figure 1. Ultrasound images during testing of the flow mediated dilation. A slightly increase of the brachial diameter from $5.44 \mathrm{~mm}$ at baseline to $5.88 \mathrm{~mm}$ under hyperemia was recorded. The Doppler flow shows an increase of the blood flow after deflation of the cuff (hyperemia). 


\section{Conclusions}

In summary, sonography is essential in the workup of a hypertensive patient. Abdominal sonography should be performed in the evaluation of hypertension. The last European guidelines have emphasized that treatment-induced changes of organ damage affect the incidence of cardiovascular events, thereby recommending performance of organ damage examinations including sonography during treatment. When a search for secondary hypertension is indicated, abdominal sonography and Doppler ultrasound of the renal arteries are also recommended. Table 2 summarizes the use of ultrasound in the evaluation of hypertension.

\begin{tabular}{|c|c|}
\hline Ultrasound examination & Use in diagnostic workup of hypertension \\
\hline Abdominal ultrasound & $\begin{array}{l}\text { Identification of subclinical and end-organ damage (recommended) } \\
\text { - Aortosclerosis } \\
\text { - Aortic aneurysm } \\
\text { - Nephrosclerosis } \\
\text { Screening for secondary causes of hypertension } \\
\text { - Renal parenchymal disease } \\
\text { - Renal vascular disease } \\
\text { - Adrenal adenomas } \\
\text { - Phaeochromocytoma }\end{array}$ \\
\hline Echocardiography & $\begin{array}{l}\text { Identification of subclinical and end organ damage (recommended) } \\
\text { - Left ventricular hypertrophy (LVH) } \\
\text { - Systolic and diastolic dysfunction } \\
\text { - Left atrium dimension and geomertry }\end{array}$ \\
\hline Doppler ultrasound of the renal arteries & $\begin{array}{l}\text { Screening for secondary causes of hypertension } \\
\text { - Renovascular hypertension }\end{array}$ \\
\hline Ultrasound of the carotid arteries & $\begin{array}{l}\text { Identification of subclinical and end-organ damage (recommended) } \\
\text { - Intima - media thickness (IMT) } \\
\text { - Carotid plaques } \\
\text { - Carotid artery stenosis }\end{array}$ \\
\hline $\begin{array}{l}\text { Assessment of flow-mediated dilation of } \\
\text { brachial artery }\end{array}$ & $\begin{array}{l}\text { Identification of subclinical organ damage (not in clinical use) } \\
\text { - Endothelial dysfunction }\end{array}$ \\
\hline
\end{tabular}

Table 2. Ultrasound examinations in hypertension

In summary, sonography is essential in the workup of a hypertensive patient. Abdominal sonography should be performed in every newly diagnosed case of hypertension. Performance of further ultrasound techniques depends on age, concomitant diseases, symptoms, and overall cardiovascular risk. Table 2 summarizes the use of ultrasound in the evaluation of hypertension. 


\section{Author details}

Nikolaos Pagonas, Stergios Vlatsas and Timm H. Westhoff

*Address all correspondence to: timm.westhoff@charite.de

Charité - Campus Benjamin Franklin, Dept. of Nephrology, Berlin, Germany

\section{References}

[1] Campos C SJ, Rodicio JL. Campos C, Segura J, Rodicio JL.. Investigations in secondary hypertension: renal disease. Hypertension. 2001:119-26.

[2] Hodis HN, Mack WJ, LaBree L, Selzer RH, Liu CR, Liu CH et al. The role of carotid arterial intima-media thickness in predicting clinical coronary events. Ann Intern Med. 1998;128(4):262-9.

[3] Rezneck RH. AP. Imaging in endocrinology. The adrenal glands.. Clin Endocrinol (Oxf). 1994;40(5):561-76.

[4] Reisch N, Peczkowska M, Januszewicz A and Neumann HP. Pheochromocytoma: presentation, diagnosis and treatment. Journal of hypertension. 2006;24(12):2331-9.

[5] Burton S. RP. Adrenal Glands. Magnetic Resonanc Imaging. 1999.

[6] Goldstein RE ONJ, Jr., Holcomb GW. Clinical experience over 48 years with pheochromocytoma. Ann Surg. 1999;229(6):755-64.

[7] Schwerk WB, Gorg C, Gorg K and Restrepo IK. Adrenal pheochromocytomas: a broad spectrum of sonographic presentation. Journal of ultrasound in medicine: official journal of the American Institute of Ultrasound in Medicine. 1994;13(7):517-21.

[8] Lloyd-Jones DM, Larson MG, Leip EP, Beiser A, D'Agostino RB, Kannel WB et al. Lifetime risk for developing congestive heart failure: the Framingham Heart Study. Circulation. 2002;106(24):3068-72.

[9] Reichek N and Devereux RB. Left ventricular hypertrophy: relationship of anatomic, echocardiographic and electrocardiographic findings. Circulation. 1981;63(6):1391-8.

[10] Mancia G, De Backer G, Dominiczak A, Cifkova R, Fagard R, Germano G et al. [ESH/ESC 2007 Guidelines for the management of arterial hypertension]. Rev Esp Cardiol. 2007;60(9):968 e1-94.

[11] Devereux RB, Wachtell K, Gerdts E, Boman K, Nieminen MS, Papademetriou V et al. Prognostic significance of left ventricular mass change during treatment of hypertension. Jama. 2004;292(19):2350-6. 
[12] Devereux RB, Alonso DR, Lutas EM, Gottlieb GJ, Campo E, Sachs I et al. Echocardiographic assessment of left ventricular hypertrophy: comparison to necropsy findings. Am J Cardiol. 1986;57(6):450-8.

[13] Muiesan ML, Salvetti M, Monteduro C, Bonzi B, Paini A, Viola S et al. Left ventricular concentric geometry during treatment adversely affects cardiovascular prognosis in hypertensive patients. Hypertension. 2004;43(4):731-8.

[14] Milani RV, Lavie CJ, Mehra MR, Ventura HO, Kurtz JD and Messerli FH. Left ventricular geometry and survival in patients with normal left ventricular ejection fraction. Am J Cardiol. 2006;97(7):959-63.

[15] Tsioufis C, Vezali E, Tsiachris D, Dimitriadis K, Taxiarchou E, Chatzis D et al. Left ventricular hypertrophy versus chronic kidney disease as predictors of cardiovascular events in hypertension: a Greek 6-year-follow-up study. J Hypertens. 2009;27(4): 744-52.

[16] Gerdts E, Wachtell K, Omvik P, Otterstad JE, Oikarinen L, Boman K et al. Left atrial size and risk of major cardiovascular events during antihypertensive treatment: losartan intervention for endpoint reduction in hypertension trial. Hypertension. 2007;49(2):311-6.

[17] Gerdts E, Cramariuc D, de Simone G, Wachtell K, Dahlof B and Devereux RB. Impact of left ventricular geometry on prognosis in hypertensive patients with left ventricular hypertrophy (the LIFE study). Eur J Echocardiogr. 2008;9(6):809-15.

[18] Aeschbacher BC, Hutter D, Fuhrer J, Weidmann P, Delacretaz E and Allemann Y. Diastolic dysfunction precedes myocardial hypertrophy in the development of hypertension. Am J Hypertens. 2001;14(2):106-13.

[19] Gotsman I, Zwas D, Lotan C and Keren A. Heart failure and preserved left ventricular function: long term clinical outcome. PLoS One. 2012;7(7):e41022.

[20] Paulus WJ, Tschope C, Sanderson JE, Rusconi C, Flachskampf FA, Rademakers FE et al. How to diagnose diastolic heart failure: a consensus statement on the diagnosis of heart failure with normal left ventricular ejection fraction by the Heart Failure and Echocardiography Associations of the European Society of Cardiology. Eur Heart J. 2007;28(20):2539-50.

[21] Moller JE, Sondergaard E, Poulsen SH and Egstrup K. Pseudonormal and restrictive filling patterns predict left ventricular dilation and cardiac death after a first myocardial infarction: a serial color M-mode Doppler echocardiographic study. J Am Coll Cardiol. 2000;36(6):1841-6.

[22] Galderisi M, Caso P, Severino S, Petrocelli A, De Simone L, Izzo A et al. Myocardial diastolic impairment caused by left ventricular hypertrophy involves basal septum more than other walls: analysis by pulsed Doppler tissue imaging. J Hypertens. 1999;17(5):685-93. 
[23] Kasner M, Westermann D, Steendijk P, Gaub R, Wilkenshoff U, Weitmann K et al. Utility of Doppler echocardiography and tissue Doppler imaging in the estimation of diastolic function in heart failure with normal ejection fraction: a comparative Doppler-conductance catheterization study. Circulation. 2007;116(6):637-47.

[24] Melenovsky V, Borlaug BA, Rosen B, Hay I, Ferruci L, Morell CH et al. Cardiovascular features of heart failure with preserved ejection fraction versus nonfailing hypertensive left ventricular hypertrophy in the urban Baltimore community: the role of atrial remodeling/dysfunction. J Am Coll Cardiol. 2007;49(2):198-207.

[25] Lang RM, Bierig M, Devereux RB, Flachskampf FA, Foster E, Pellikka PA et al. Recommendations for chamber quantification. Eur J Echocardiogr. 2006;7(2):79-108.

[26] Lang RM, Bierig M, Devereux RB, Flachskampf FA, Foster E, Pellikka PA et al. Recommendations for chamber quantification: a report from the American Society of Echocardiography's Guidelines and Standards Committee and the Chamber Quantification Writing Group, developed in conjunction with the European Association of Echocardiography, a branch of the European Society of Cardiology. J Am Soc Echocardiogr. 2005;18(12):1440-63.

[27] Gopal AS, Schnellbaecher MJ, Shen Z, Boxt LM, Katz J and King DL. Freehand threedimensional echocardiography for determination of left ventricular volume and mass in patients with abnormal ventricles: comparison with magnetic resonance imaging. J Am Soc Echocardiogr. 1997;10(8):853-61.

[28] Buck T, Hunold P, Wentz KU, Tkalec W, Nesser HJ and Erbel R. Tomographic threedimensional echocardiographic determination of chamber size and systolic function in patients with left ventricular aneurysm: comparison to magnetic resonance imaging, cineventriculography, and two-dimensional echocardiography. Circulation. 1997;96(12):4286-97.

[29] Cuspidi C, Ambrosioni E, Mancia G, Pessina AC, Trimarco B and Zanchetti A. Role of echocardiography and carotid ultrasonography in stratifying risk in patients with essential hypertension: the Assessment of Prognostic Risk Observational Survey. J Hypertens. 2002;20(7):1307-14.

[30] Schillaci G, De Simone G, Reboldi G, Porcellati C, Devereux RB and Verdecchia P. Change in cardiovascular risk profile by echocardiography in low- or medium-risk hypertension. J Hypertens. 2002;20(8):1519-25.

[31] Elliott W. Secondary hypertension: renovascular hypertension. Hypertension: a Companion to Braunwald's Heart Disease. 2007:93-105.

[32] Radermacher J, Chavan A, Schaffer J, Stoess B, Vitzthum A, Kliem V et al. Detection of significant renal artery stenosis with color Doppler sonography: combining extrarenal and intrarenal approaches to minimize technical failure. Clinical nephrology. 2000;53(5):333-43. 
[33] Simoni C, Balestra G, Bandini A and Rusticali F. [Doppler ultrasound in the diagnosis of renal artery stenosis in hypertensive patients: a prospective study]. Giornale italiano di cardiologia. 1991;21(3):249-55.

[34] Safian RD and Textor SC. Renal-artery stenosis. N Engl J Med. 2001;344(6):431-42.

[35] Radermacher J and Brunkhorst R. Diagnosis and treatment of renovascular stenosis-a cost-benefit analysis. Nephrology, dialysis, transplantation: official publication of the European Dialysis and Transplant Association - European Renal Association. 1998;13(11):2761-7.

[36] Schwerk WB, Restrepo IK, Stellwaag M, Klose KJ and Schade-Brittinger C. Renal artery stenosis: grading with image-directed Doppler US evaluation of renal resistive index. Radiology. 1994;190(3):785-90.

[37] Krumme B. Renal Doppler sonography--update in clinical nephrology. Nephron. Clinical practice. 2006;103(2):c24-8.

[38] Pearce JD, Edwards MS, Craven TE, English WP, Mondi MM, Reavis SW et al. Renal duplex parameters, blood pressure, and renal function in elderly people. American journal of kidney diseases: the official journal of the National Kidney Foundation. 2005;45(5):842-50.

[39] Radermacher J, Chavan A, Bleck J, Vitzthum A, Stoess B, Gebel MJ et al. Use of Doppler ultrasonography to predict the outcome of therapy for renal-artery stenosis. $\mathrm{N}$ Engl J Med. 2001;344(6):410-7.

[40] Zanchetti A, Bond MG, Hennig M, Neiss A, Mancia G, Dal Palu C et al. Calcium antagonist lacidipine slows down progression of asymptomatic carotid atherosclerosis: principal results of the European Lacidipine Study on Atherosclerosis (ELSA), a randomized, double-blind, long-term trial. Circulation. 2002;106(19):2422-7.

[41] Bots ML, Hoes AW, Koudstaal PJ, Hofman A and Grobbee DE. Common carotid intima-media thickness and risk of stroke and myocardial infarction: the Rotterdam Study. Circulation. 1997;96(5):1432-7.

[42] Lorenz MW, Markus HS, Bots ML, Rosvall M and Sitzer M. Prediction of clinical cardiovascular events with carotid intima-media thickness: a systematic review and meta-analysis. Circulation. 2007;115(4):459-67.

[43] Mansia G, De Backer G, Dominiczak A, Cifkova R, Fagard R, Germano G et al. 2007 ESH-ESC Guidelines for the management of arterial hypertension: the task force for the management of arterial hypertension of the European Society of Hypertension (ESH) and of the European Society of Cardiology (ESC). Blood Press. 2007;16(3): 135-232.

[44] Stein JH, Korcarz CE, Hurst RT, Lonn E, Kendall CB, Mohler ER et al. Use of carotid ultrasound to identify subclinical vascular disease and evaluate cardiovascular disease risk: a consensus statement from the American Society of Echocardiography 
Carotid Intima-Media Thickness Task Force. Endorsed by the Society for Vascular Medicine. J Am Soc Echocardiogr. 2008;21(2):93-111; quiz 89-90.

[45] Wong M, Edelstein J, Wollman J and Bond MG. Ultrasonic-pathological comparison of the human arterial wall. Verification of intima-media thickness. Arterioscler Thromb. 1993;13(4):482-6.

[46] Touboul PJ, Hennerici MG, Meairs S, Adams H, Amarenco P, Bornstein N et al. Mannheim carotid intima-media thickness consensus (2004-2006). An update on behalf of the Advisory Board of the 3rd and 4th Watching the Risk Symposium, 13th and 15th European Stroke Conferences, Mannheim, Germany, 2004, and Brussels, Belgium, 2006. Cerebrovasc Dis. 2007;23(1):75-80.

[47] Paini A, Boutouyrie P, Calvet D, Zidi M, Agabiti-Rosei E and Laurent S. Multiaxial mechanical characteristics of carotid plaque: analysis by multiarray echotracking system. Stroke. 2007;38(1):117-23.

[48] EA Rosei MM. Assessment of preclinical target organ damage in hypertension: carotid intima-media thickness and plaque. European Society of Hypertension Schientific Newsletter. 2011pp. 19-20.

[49] Zanchetti A, Hennig M, Hollweck R, Bond G, Tang R, Cuspidi C et al. Baseline values but not treatment-induced changes in carotid intima-media thickness predict incident cardiovascular events in treated hypertensive patients: findings in the European Lacidipine Study on Atherosclerosis (ELSA). Circulation. 2009;120(12): 1084-90.

[50] Sehestedt T, Jeppesen J, Hansen TW, Rasmussen S, Wachtell K, Ibsen H et al. Which markers of subclinical organ damage to measure in individuals with high normal blood pressure? J Hypertens. 2009;27(6):1165-71.

[51] Salonen JT and Salonen R. Ultrasonographically assessed carotid morphology and the risk of coronary heart disease. Arterioscler Thromb. 1991;11(5):1245-9.

[52] Werner GS, Fritzenwanger M, Prochnau D, Schwarz G, Ferrari M, Aarnoudse W et al. Determinants of coronary steal in chronic total coronary occlusions donor artery, collateral, and microvascular resistance. J Am Coll Cardiol. 2006;48(1):51-8.

[53] Zanchetti A, Crepaldi G, Bond MG, Gallus G, Veglia F, Mancia G et al. Different effects of antihypertensive regimens based on fosinopril or hydrochlorothiazide with or without lipid lowering by pravastatin on progression of asymptomatic carotid atherosclerosis: principal results of PHYLLIS--a randomized double-blind trial. Stroke. 2004;35(12):2807-12.

[54] Chalmers J, Todd A, Chapman N, Beilin L, Davis S, Donnan G et al. International Society of Hypertension (ISH): statement on blood pressure lowering and stroke prevention. J Hypertens. 2003;21(4):651-63.

[55] Collins R, Peto R, MacMahon S, Hebert P, Fiebach NH, Eberlein KA et al. Blood pressure, stroke, and coronary heart disease. Part 2, Short-term reductions in blood pres- 
sure: overview of randomised drug trials in their epidemiological context. Lancet. 1990;335(8693):827-38.

[56] Rodgers A, MacMahon S, Gamble G, Slattery J, Sandercock P and Warlow C. Blood pressure and risk of stroke in patients with cerebrovascular disease. The United Kingdom Transient Ischaemic Attack Collaborative Group. Bmj. 1996;313(7050):147.

[57] Wardlaw JM, Chappell FM, Best JJ, Wartolowska K and Berry E. Non-invasive imaging compared with intra-arterial angiography in the diagnosis of symptomatic carotid stenosis: a meta-analysis. Lancet. 2006;367(9521):1503-12.

[58] Guidelines for management of ischaemic stroke and transient ischaemic attack 2008. Cerebrovasc Dis. 2008;25(5):457-507.

[59] Grant EG, Duerinckx AJ, El Saden SM, Melany ML, Hathout GM, Zimmerman PT et al. Ability to use duplex US to quantify internal carotid arterial stenoses: fact or fiction? Radiology. 2000;214(1):247-52.

[60] Brott TG, Halperin JL, Abbara S, Bacharach JM, Barr JD, Bush RL et al. 2011 ASA/ ACCF/AHA/AANN/AANS/ACR/ASNR/CNS/SAIP/SCAI/SIR/SNIS/SVM/SVS guideline on the management of patients with extracranial carotid and vertebral artery disease: executive summary. J Neurointerv Surg.3(2):100-30.

[61] Celermajer DS, Sorensen KE, Gooch VM, Spiegelhalter DJ, Miller OI, Sullivan ID et al. Non-invasive detection of endothelial dysfunction in children and adults at risk of atherosclerosis. Lancet. 1992;340(8828):1111-5.

[62] Iiyama K, Nagano M, Yo Y, Nagano N, Kamide K, Higaki J et al. Impaired endothelial function with essential hypertension assessed by ultrasonography. Am Heart J. 1996;132(4):779-82.

[63] Panza JA, Quyyumi AA, Brush JE, Jr. and Epstein SE. Abnormal endothelium-dependent vascular relaxation in patients with essential hypertension. $\mathrm{N}$ Engl J Med. 1990;323(1):22-7.

[64] Muiesan ML, Salvetti M, Monteduro C, Rizzoni D, Zulli R, Corbellini C et al. Effect of treatment on flow-dependent vasodilation of the brachial artery in essential hypertension. Hypertension. 1999;33(1 Pt 2):575-80.

[65] Kathiresan S, Gona P, Larson MG, Vita JA, Mitchell GF, Tofler GH et al. Cross-sectional relations of multiple biomarkers from distinct biological pathways to brachial artery endothelial function. Circulation. 2006;113(7):938-45.

[66] Juonala M, Viikari JS, Alfthan G, Marniemi J, Kahonen M, Taittonen L et al. Brachial artery flow-mediated dilation and asymmetrical dimethylarginine in the cardiovascular risk in young Finns study. Circulation. 2007;116(12):1367-73.

[67] Corretti MC, Anderson TJ, Benjamin EJ, Celermajer D, Charbonneau F, Creager MA et al. Guidelines for the ultrasound assessment of endothelial-dependent flow-medi- 
ated vasodilation of the brachial artery: a report of the International Brachial Artery Reactivity Task Force. J Am Coll Cardiol. 2002;39(2):257-65. 
